\title{
Fluctuation spectroscopy of surface melting of ice without, and with impurities
}

\author{
Takahisa Mitsui* and Kenichiro Aoki ${ }^{\dagger}$ \\ Research and Education Center for Natural Sciences and Dept. of Physics, \\ Hiyoshi, Keio University, Yokohama 223-8521, Japan
}

\begin{abstract}
Water is ubiquitous, and surface properties of ice has been studied for some time, due to its importance. Liquid-like layer (LLL) is known to exist on ice, below the melting point. We use surface thermal fluctuation spectroscopy to study LLL, including its thickness, for pure ice, and for ice with impurities. We find that the properties of LLL are experimentally those of liquid water, with thickness much smaller than previous results. We also find that impurities cause LLL to be thicker, and be quite inhomogeneous, with properties depending on the dopant.
\end{abstract}

The importance of understanding the surface properties of ice in air can not be understated, which is crucial for the clarification of the melting, freezing process itself, as well being important to a broad range fields in science [1-3]. Theoretically, the existence of LLL (also called "quasi-liquid layer", or QLL) has been discussed since 19th century, and its properties, including the existence, has been studied from various points of view - thermodynamic arguments [4 [6], and more recently, state of the art molecular dynamics simulations have been employed [-15]. Due to its importance, and its experimental difficulty, surface melting of ice has been analyzed experimentally using a multitude of methods, such as ellipsometry 16 18], X-ray diffraction 19], proton backscattering [20], photoelectron spectroscopy 21], atomic force microscopy 22 24, and surface sumfrequency generation (SFG) spectroscopy 25 27]. For a number of reasons, including the thinness of LLL, which can be $\mathrm{nm}$ order or smaller, the study of LLL remains to be an experimentally challenging problem. This difficulty is evidenced by the thickness measurements of LLL of ice, which vary by orders of magnitude [3, 18, 28].

In this work, we optically measure the surface thermal fluctuations of LLL on ice, in air. By measuring the thermal motions of the molecules directly, in addition to clearly differentiating the liquid and solid phases, the properties of the material become apparent 29 34]. Such a method, while not previously applied to surface melting, has proven to be effective in understanding the properties of various liquids, complex fluids, and viscoelastic materials 35 40]. This experimental method for measuring the properties of LLL, distinct from previous methods, enables us to obtain a different perspective on LLL. The surface thermal fluctuations, which are spontaneous, reveal the properties of the material underneath, providing information whether LLL has the properties of water in the bulk, in addition to the behavior of its thickness. The importance of thermal fluctuations of the LLL were recognized, and their properties were recently analyzed

\footnotetext{
${ }^{*}$ E-mail: mitsui@phys-h.keio.ac.jp.

${ }^{\dagger} \mathrm{E}-\mathrm{mail}$ : ken@phys-h.keio.ac.jp.
}

using simulations [41]. There, it was found that the surface fluctuations are similar to those of water-vapor interfaces, with the fluctuation spectra strongly affected by the thickness of LLL, consistent with our results. Also, it has been found using SFG spectroscopy 25 27], that the surface of LLL behaves similarly to bulk supercooled water, seemingly consistent with our results. We find that LLL is much thinner than most of the previous experimental results, and that the additions of impurities at ppm levels thicken LLL. Furthermore, by scanning the surface at $\mu \mathrm{m}$ level resolution, we directly observe that impurities cause inhomogeneities, with their properties depending on the dopant.

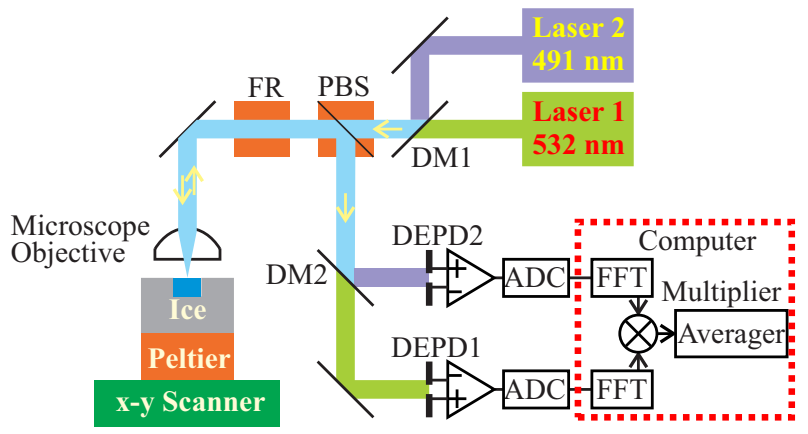

FIG. 1: Experimental setup: Linearly polarized laser light with wavelengths $532,491 \mathrm{~nm}$ are shone on the sample ice surface, with powers $340,270 \mu \mathrm{W}$ at the surface. The reflected light is directed to two dual-element photodiodes (DEPD1,2) corresponding to Laser 1,2 . The differences in the light beam powers in the DEPDs are digitized using analog-to-digital converters (ADC), Fourier transformed (FFT), and the averaged correlation is computed (averager). Faraday rotator (FR) is used to rotate the polarization of the light by $\pi / 4$, each way, so that the polarized beam splitter (PBS) reflects the light from the sample. Dichroic mirrors (DM1,2) are used to merge, and separate the light with different wavelengths.

The experimental setup is shown in Fig. 1] Light is shone on the surface of ice, with LLL expected to be on it. The reflected light is detected by the dual-element photodiode (DEPD). The surface acts as a partial mirror, and the two elements in DEPD produce the same photocurrent, if the surface is not fluctuating. The surface fluctuates thermally, and produces fluctuations in 
the photocurrent difference, whose power spectrum is the inclination fluctuation spectrum of the surface, up to a constant. The measurement is performed using two light sources with different wavelengths, in order to use correlation analysis to statistically reduce the extraneous noise [37], to orders of magnitude below the shotnoise level, often referred to as the "Standard Quantum Limit" 42]. The beam radius (waist) at the sample, $w$, is $1.2 \mu \mathrm{m}$, and the sample can be moved horizontally in two dimensions by $15 \mu \mathrm{m}$ in each direction, allowing us to scan the surface. Recently, LCM-DIM (laser confocal microscopy combined with differential contrast microscopy) has been developed to optically observe ice surfaces [43, 44]. Our method measures the thickness of LLL, which is not measurable in LCM-DIM. LCM-DIM has been used to measure transient properties of surface structures, in contrast to our thermal equilibrium measurements, and while both methods are optical measurements, we find these methods complementary. Due to the breadth of interest, importance of the surface melting phenomena, and the difficulties in measuring their properties, various experimental approaches have been brought to bear on this problem. We believe that it is important to investigate the phenomena from different perspectives, both experimental and theoretical, to clarify the inner workings underlying them. One important character of our approach is that it makes optical measurements of only the spontaneous surface fluctuations, with no external stimulation, and is hence minimally invasive. The time-scales for our measurements at relatively longer time scales, at least order of tens of seconds. The approach is suited for measurements of thermal equilibrium properties, and not for observing transient effects. The measurements made in this work are stable on the order of hours.

The liquid sample to be frozen was put into the stainless steel container (diameter $3.5 \mathrm{~mm}$, depth $1.5 \mathrm{~mm}$ ), and thermoelectrically cooled. Due to supercooling, the liquid froze at around $-20^{\circ} \mathrm{C}$, without, and with impurities. For pure water, and $\mathrm{NaCl}$ solution cases, the temperature of the liquid was then raised to $0.07^{\circ} \mathrm{C}$, and kept at that temperature till the water layer on ice was about $0.5 \mathrm{~mm}$, which took around 30 minutes. Next, the sample liquid temperature was lowered gradually to $-0.03^{\circ} \mathrm{C}$, taking over an hour. After this, the measurements were then taken, at various temperatures. For the Volvic sample, the same procedure led to too much precipitation of minerals, leading to unwanted noise in the measurement due to the unevenness of the surface. Therefore, to reduce the precipitation, the temperature of the sample was raised to to $-2.4^{\circ} \mathrm{C}$ after freezing the supercooled Volvic water, and kept constant for over an hour, after which measurements at various temperatures were taken. Water sample was purified using the EMD Millipore Purification System (Merck Millipore, Germany).

Our experimental setup measures the thermal fluctua-

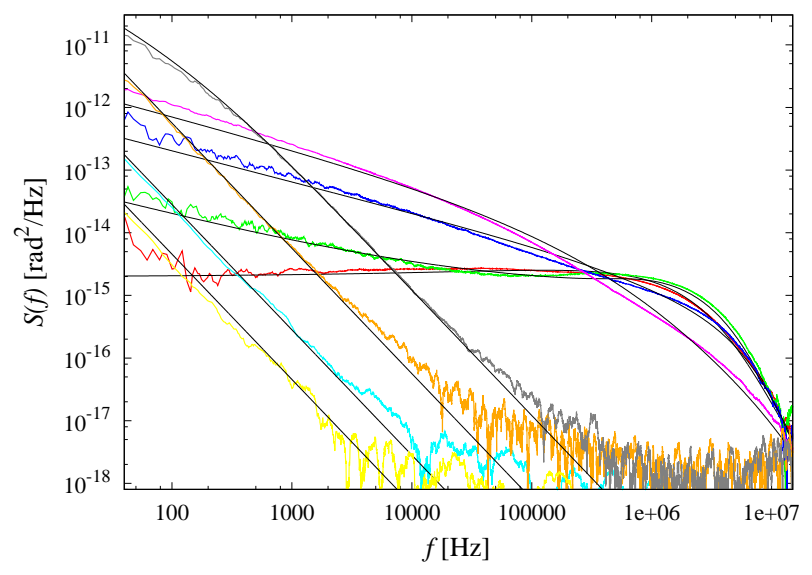

FIG. 2: Surface thermal fluctuation spectra of water layer of various thicknesses: Fluctuation spectra for supercooled water $(\Delta T=17.89 \mathrm{~K}$, red $)$, and LLL with $(\Delta T[\mathrm{~K}], h[\mathrm{~m}])=\left(0.003,3.0 \times 10^{-6}\right)($ green $),(0.004,6.0 \times$ $\left.10^{-7}\right)$ (blue), $\left(0.006,2.5 \times 10^{-7}\right)$ (magenta), $\left(0.014,1.0 \times 10^{-8}\right)$ (grey), $\left(0.024,3.6 \times 10^{-9}\right)$ (orange), $\left(1.39,1.4 \times 10^{-9}\right)($ cyan $)$, $\left(24.89,1.0 \times 10^{-9}\right)$ (yellow). Corresponding theoretical spectra for water with finite depth are also shown(black), and agree well with the measured spectra.

tion spectra of the averaged inclination within the beam spot on the sample 37,

$$
S(f)=\int_{0}^{\infty} d k k^{3} e^{-w^{2} k^{2} / 4} F(k, f, h)
$$

Here, $f$ is the frequency, and $F(k, f, h)$ is the spectral function of the thermal fluctuations of the fluid surface, and depends on LLL thickness, $h[32$. The spectrum is uniquely determined by the bulk properties of water (density, surface tension, viscosity), beam size, $h$, and the sample temperature. Gravitational effects are negligible at our sample size. Some examples of spectra for LLL, and water at various $h, \Delta T=T_{\mathrm{m}}-T$ are shown in Fig. 2, $T$ is the temperature of the sample surface, and $T_{\mathrm{m}}$ is the bulk melting temperature of ice. It can be seen that the spectral shape depends strongly on $h$, and the experimental spectra agree quite well with the theoretical spectra of surface thermal fluctuations of water with finite thickness. The known bulk properties of supercooled water 45 48 were used to compute the theoretical spectra. We note that surface thermal fluctuations of solid ice without LLL should not only be much smaller, but behave as $1 / f[36,37]$, which is incompatible with the measurements. For LLL with $h \gtrsim 10 \mathrm{~nm}$, the spectral shape and the magnitude are sensitive to the fluid properties of LLL, density, surface tension, and viscosity, as well as its thickness, in our experimental setting. In particular, in all the spectra analyzed, the viscosity inferred from the spectrum is consistent with the known physical properties of supercooled water. For $h \lesssim 10 \mathrm{~nm}$, the 
theoretical spectrum behaves as

$$
S(f) \simeq \frac{16}{3 \pi^{3}} \frac{k_{\mathrm{B}} T}{\eta w^{6}} \frac{h^{3}}{f^{2}},
$$

where $\eta$ is the viscosity of (supercooled) water, $k_{\mathrm{B}}$ is the Boltzmann constant, and $T$ is the temperature. This $1 / f^{2}$ behavior is indeed observed for thinner LLL in Fig. 2. While the spectral magnitude is quite sensitive to the thickness, the spectral shape is rather insensitive to the properties of LLL.

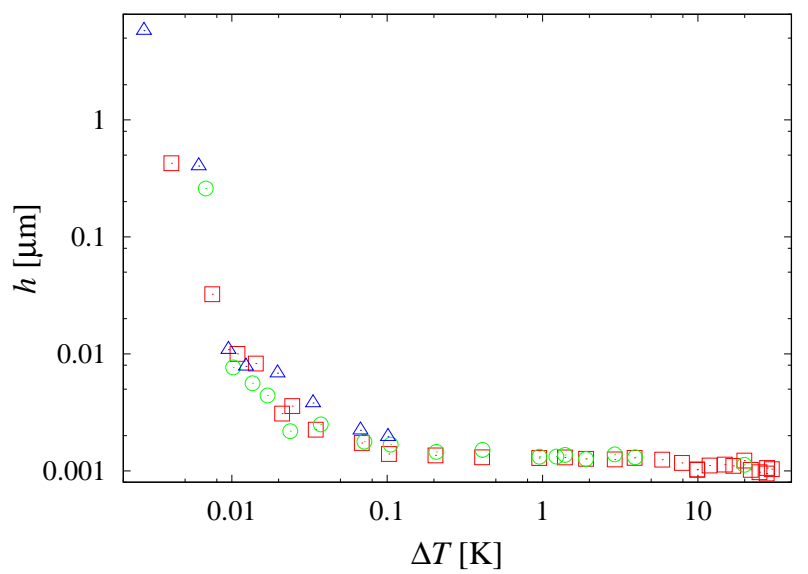

FIG. 3: The temperature dependence of the thickness of LLL for pure ice: The three types of points $(\bigcirc, \triangle, \square)$ correspond to three data sets taken with three different samples, on three different days, which are seen to be consistent within error.

The dependence of $h$ for LLL of pure ice on the temperature is shown in Fig. 3. $h$ was estimated from the surface thermal fluctuation spectra (Fig. 21): For $h \gtrsim 10 \mathrm{~nm}$, theoretical spectral shape was fit to the measurements, and for smaller $h$, the measured spectrum was normalized using the shot-noise level. The relation between $h$ and $\Delta T$ has been studied previously by a number of authors, using various other methods, and $h$ differs by orders of magnitude, depending on the method used [3, 18, 28]. Our results extend over a much wider range of temperatures than that previously covered with any one method. The results in Fig. 3 are consistent between measurements of different samples taken on different days, and also between measurements taken with the temperatures in ascending, and descending sequences. This strongly suggests that the results are equilibrium properties. Our results for $h$ are smaller than those previously measured for $\Delta T<1 \mathrm{~K}$, close to the bulk melting temperature. Previous results are almost non-existent for $\Delta T>10 \mathrm{~K}$, except for photoelectron emission spectroscopy study of LLL of ice in pure water vapor 21]. Compared with this, our results for $h$ are of the same order but slightly larger in this temperature range. On the theoretical side, some thermodynamic considerations predict much thicker [5], and also thinner [6] LLL. Recent molecular dynamics simulation results exist for $\Delta T \gtrsim 1 \mathrm{~K}[9,12,13,15,41]$, with thicknesses smaller than ours, but consistent within a factor of few. It should be noted that previous literature with thinner LLL, both theoretical and experimental [6, 9, 13, 21], deal with ice in pure water vapor, without air, unlike our results, which might be the reason for the difference. For thin layers, viscosity might be larger than its value in bulk, though whether it is, and by how much if so, is an unsettled question 2, 49 51]. Since the surface thermal fluctuation spectrum behaves as $\sim h^{3} /\left(\eta f^{2}\right)$ for thin LLL, larger viscosities lead to larger $h$ values in our results (Fig. 3), so that a dramatic rise in the viscosity for smaller $h$ seems unlikely.

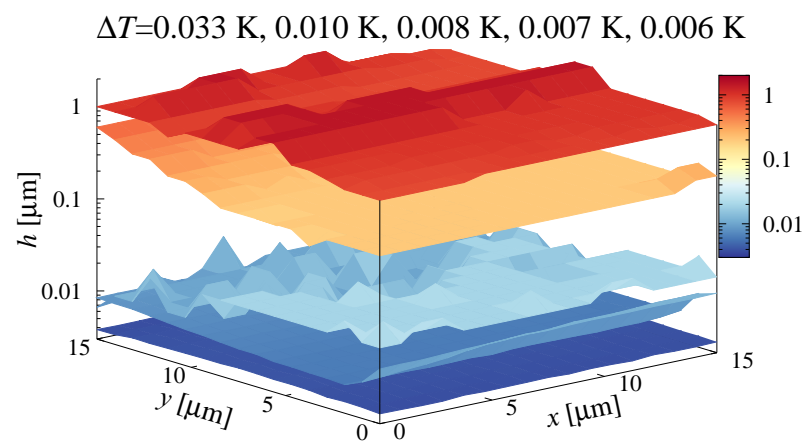

FIG. 4: Spatial distribution of LLL thickness of pure ice: $h$, at four temperatures, $\Delta T=$ $0.033,0.010,0.008,0.007,0.006 \mathrm{~K} . \quad h$ is larger at lower temperatures (larger $\Delta T$ ). $h$ distributions are seen to be relatively uniform, with slight variations.

An important questions is whether LLL is homogeneous: Our measurement system allows for scanning at $\mu m$ level, since the light beam is focused, with $w=$ $1.2 \mu \mathrm{m}$. In Fig. 4 the dependence of the $h$ on the surface location is shown, for the surface of pure ice, at different temperatures. $h$ values are seen to be reasonably uniform in the scanned region, for LLL of pure ice. For small $\Delta T, h$ is strongly dependent on the temperature (Fig. 3), so some variation is visible. Molecular dynamics simulations exist that suggest the transition from liquid to solid is not sharp for LLL 15, 41]. The properties we found through the surface thermal fluctuations are consistent with a thin layer having the properties of bulk water, as seen in Fig. 2. We expect this not to preclude a gradual transition from the liquid-like to the solid-like structure at the bottom, as long as waves with amplitudes larger than those at the liquid-gas interface do not propagate in this transition layer. It would be interesting to investigate if more precise measurements, and further information can be extracted from surface thermal fluctuation measurements, especially when LLL is thin, around $1 \mathrm{~nm}$ or less. Thermodynamic arguments combined with microscopic simulation suggest, interestingly, that the ice-liquid interface has a roughness, at atomic scales [52]. However, since our measurements are aver- 
aged within the beam at the $\mu \mathrm{m}$ order, such roughness is not observable.
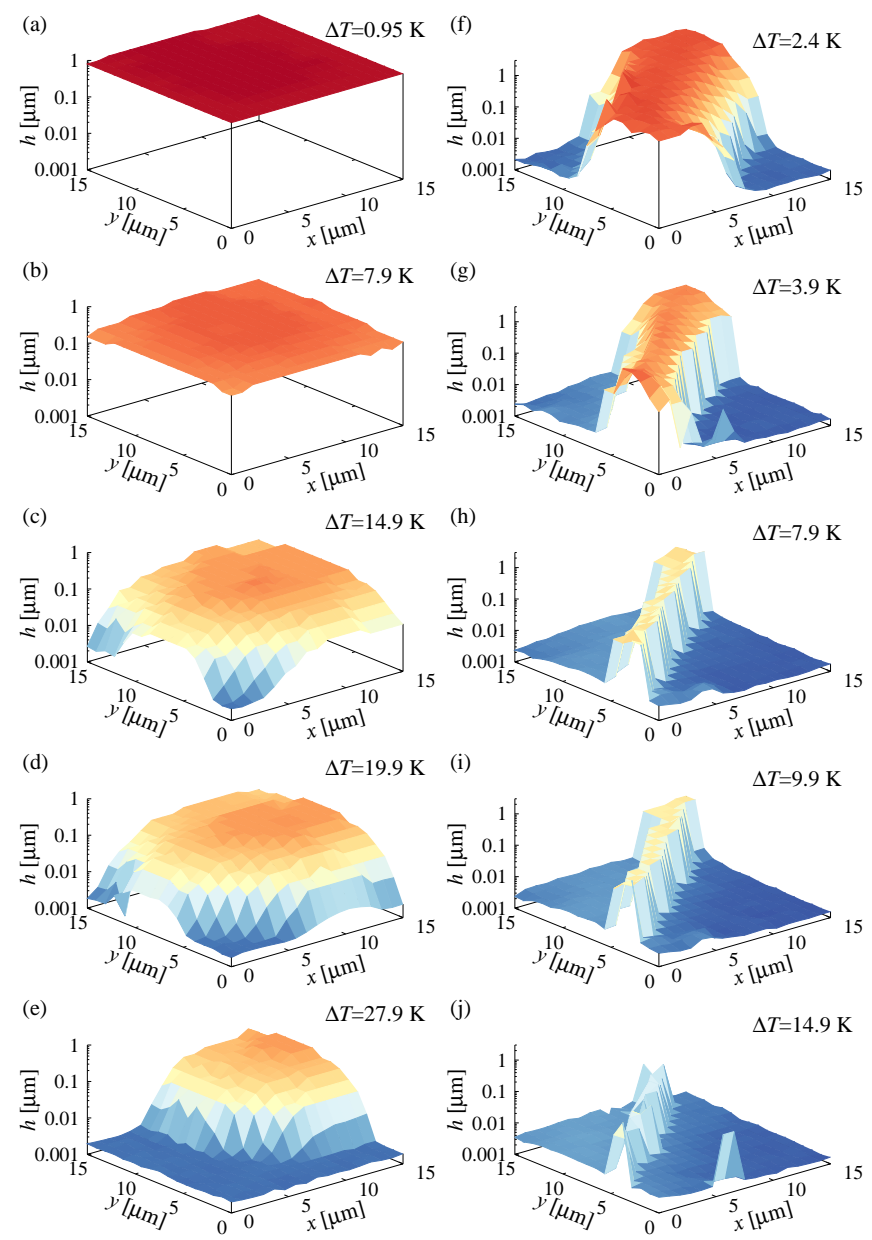

FIG. 5: Spatial distribution of $h$ for ice with $\mathrm{NaCl}((\mathrm{a})-(\mathrm{e}))$, and Volvic $((\mathrm{f})-(\mathrm{j}))$, as the temperature is lowered, for the same area.

Theoretically, impurities can greatly affect the overall thickness of LLL [53, 54], and are perhaps the major cause of their large observed disparities [2]. To study the effect of impurities, in Fig. 5, the spatial dependences of $h$ are shown for frozen $\mathrm{NaCl}$ solution (10 ppm by weight before freezing), and Volvic (water with minerals roughly $60 \mathrm{ppm}$ by weight) [55] at various temperatures, as they are being cooled. Such direct observations of the inhomogeneities have been observed for the first time. We chose Volvic, which contains various minerals, as a model of water in a natural setting. There are clear qualitative differences from the properties of LLL of pure ice, and also between LLL with different impurities. First, we see that in both cases, LLL with $h>0.1 \mu \mathrm{m}$ exists for $\Delta T>1 \mathrm{~K}$, in contrast to that of pure ice, seen in Fig. 3. Furthermore, unlike pure ice LLL, $h$ distributions are quite inhomogeneous. There is also a distinct difference between the effect of two impurities: A reasonably thick LLL exists for frozen $\mathrm{NaCl}$ solution down to temperatures much lower than that for Volvic, which is not as inhomogeneous as the latter. For Volvic, channels of LLL form, which have the bulk properties of water, that grow narrower and shallower as the temperature lowers. Interestingly, vein like structures have been observed in glaciers [2, 56]. The cause for the distinct difference between ice with $\mathrm{NaCl}$ and Volvic can perhaps be attributed to the difference in the solubility of the impurities. Minerals within Volvic are not as soluble in water as $\mathrm{NaCl}$, in general, probably leading to more inhomogeneities and precipitation. We have scanned the surface of ice with glucose, and have found that the behavior is similar to that of $\mathrm{NaCl}$ solution. Results for ice with $\mathrm{NaCl}$ at various concentrations, and frozen Evian water [57], and are also consistent with the above considerations. The concentration per unit area of the $\mathrm{NaCl}$ solution in Fig. 5 can be estimated to be $90 \mu \cdot \mathrm{mol} / \mathrm{m}^{2}$. The observed thicknesses of LLL seem roughly consistent with those of the theory [53], though at lower temperatures, the inhomogeneity of LLL should be taken into account.

An interesting classic problem is the ability to skate on ice, or the slipperiness of ice. The most classic explanation for its cause is pressure melting. However the theory is insufficient in explaining the ability to skate, except very close to the melting point, due to the pressures required [2]. One explanation is the existence of a relatively thick LLL. We found that pure ice only supports LLL with thicknesses under $1 \mathrm{~nm}$ for $\Delta T>1 \mathrm{~K}$. However, in a natural setting, water inevitably contains a certain amount of impurities. Our results show that impurities thicken LLL, and create inhomogeneities, which can contribute to the slipperiness of ice. One observation from our results is that frozen mineral water supports surface melting in the form of veins, which essentially disappear for temperatures below $-10^{\circ} \mathrm{C}$. This is intriguing considering that optimal skating conditions are considered to be between -5 to $-9^{\circ} \mathrm{C}[58]$. Frictional heating [2], and other mechanisms have also been recently suggested [44, 59], and further investigation remains.

[1] H.H. Jellinek, J. Coll. Int. Sci. 25, 192(1967).

[2] J.G. Dash, A.W. Rempel, J.S. Wettlaufer, Rev. Mod. Phys. 78, 695(2006).

[3] T. Bartels-Rausch, et al., Atm. Chem. Phys. 14, 1587(2014).

[4] W.A. Weyl, J. Coll. Sci 6, 389(1951).

[5] N.H. Fletcher, Phil. Mag. 7, 255(1962); 18, 1287(1968).

[6] M. Elbaum, M. Schick, Phys. Rev. Lett. 66, 1713(1991).

[7] G.J. Kroes, Surf. Sci. 275, 365(1992).

[8] H. Nada, Y. Furukawa, Surf. Sci. 446, 1(2000).

[9] M.M. Conde, C. Vega, A. Patrykiejew, J. Chem. Phys. 129, 014702 (2008).

[10] T. Ikeda-Fukazawa, K. Kawamura, J. Chem. Phys. 120, 1395 (2004).

[11] W. Pfalzgraff, William, S. Neshyba, M. Roeselova, J. 
Phys. Chem. A115, 6184(2011).

[12] T.D. Shepherd, M.A. Koc, V. Molinero, J. Phys. Chem. C116, 12172 (2012).

[13] D.T. Limmer, D. Chandler, J. Chem. Phys. 141, 18C505 (2014).

[14] A. Mohandesi, P.G. Kusalik, J. Cryst. Growth 483, 156 (2018).

[15] I. Pickering, M. Paleico, Y.A.P. Serkin, D.A. Scherlis, M.H. Factorovich, J. Phys. Chem. B122, 4880(2018).

[16] D. Beaglehole, D. Nason, Surf. Sci. 96, 357(1980).

[17] Y. Furukawa, M. Yamamoto, T. Kuroda, J. Cryst. Growth 82, 665(1987).

[18] Y. Furukawa, H. Nada, J. Phys. Chem. B101, 6167(1997).

[19] A. Lied, H. Dosch, J.H. Bilgram, Physica B 198, 92(1994).

[20] I. Golecki, C. Jaccard, J. Phys. C11, 4229(1978).

[21] H. Bluhm, D.F. Ogletree, C.S. Fadley, Z. Hussain, N. Salmeron, J. Phys. Cond. Matt. 14, L227(2002).

[22] A. Döppenschmidt, H.J. Butt, Langmuir 16, 6709(2000).

[23] B. Pittenger et al. Phys. Rev. B 63, 134102 (2001).

[24] M.P. Goertz, X.-Y. Zhu, J.E. Houston, Langmuir 25, 6905(2009).

[25] M.A. Sanchez et al., Proc. Nat. Acad. Sci. 114, 227(2017).

[26] W.J. Smit et al., Phys. Rev. Lett. 119, 133003 (2017).

[27] W.J. Smit, H.J. Bakker, Angew. Chem. 129, 1574615750 (2017).

[28] M. Elbaum, S.G. Lipson, J.G. Dash, J. Cryst. Growth 129, 491(1993).

[29] V.G. Levich, "Physicochemical Hydrodynamics", Prentice-Hall, Englewood Cliffs (1962).

[30] P. Cicuta, I. Hopkinson, Coll. Surf. A233, 97(2004).

[31] M.A. Bouchiat, J. Meunier, J. de Phys. 32, 561(1971).

[32] J. Jäckle, J. Phys. Cond. Matt. 10, 7121(1998).

[33] D. Langevin, (Ed), "Light scattering by liquid surfaces and complementary techniques", Marcel Dekker, New York (1992).

[34] L.M.C. Sagis, Rev. Mod. Phys. 83, 1367(2011).

[35] T. Mitsui, Jpn. J. Appl. Phys. , 44, 3279(2005).

[36] A. Tay et al., Rev. Sci. Instrum. 79, 103107 (2008).

[37] T. Mitsui, K. Aoki, Phys. Rev. E 80, 020602(R) (2009).

[38] B. Pottier, G. Ducouret, C. Frétigny, F. Lequeux, L. Tal- ini, Soft Matter, 7, 7843(2011).

[39] B. Pottier, C. Fretigny, L. Talini, Phys. Rev. Lett. 114, 227801 (2015).

[40] K. Aoki, T. Mitsui, Prog. Theor. Exp. Phys. 2018, 043J01 (2018).

[41] J. Benet, P. Llombart, E. Sanz, L.G. MacDowell, Phys. Rev. Lett. 117, 096101 (2016).

[42] Walls, D.F., Milburn, G.J. "Quantum Optics", Springer, Heidelberg (2008).

[43] H. Asakawa, G. Sazaki, K. Nagashima, S. Nakatsubo, Y. Furukawa, Proc. Nat. Acad. Sci. 113, 1749(2016).

[44] K. Murata, H. Asakawa, K. Nagashima, Y. Furukawa, G. Sazaki, Proc. Nat. Acad. Sci. 113, E6741(2016).

[45] L.D. Eicher, B.J. Zwolinski, J. Phys. Chem. 75, 2016(1971).

[46] R.J. Speedy, J. Phys. Chem. 12, 3354(1987).

[47] V. Vins, M. Fransen, J. Hykl, J. Hruby, J. Phys. Chem. B119, 5567(2015).

[48] W.M. Haynes, "CRC Handbook of Chemistry and Physics, 92nd Edition", CRC Press (Baton Rouge, 2011).

[49] S.S. Barer, N.V. Churaev, B.V. Derjaguin, O.A. Kiseleva, V.D. Sobolev, J. Coll. Int. Sci. 74, 173(1980).

[50] S. Mantovani, S. Valeri, A. Loria, U. Delpennino, J. Chem Phys. 72, 1077(1980).

[51] S.A. Bardasov, V.D. Sobolev, N.V. Churaev, Coll. J. Russ. Acad. Sci. 55, 512(1993).

[52] Y. Qiu, V. Molinero, J. Phys. Chem. Lett. 9, 5179(2018).

[53] J.S. Wettlaufer, Phys.Rev. Lett. 82, 2516(1999).

[54] L. Benatov, J. S. Wettlaufer, Phys. Rev. E 70, 061606 (2004).

[55] https://www.danone.com/brands/waters/volvic.html

[56] C.F. Raymond, W.D. Harrison, J. Glaciol. 14, 213(2017).

[57] http://www.evian.com.

[58] http://www . eyeontheice.com/documents/olympic\%20ice\%20making [59] B. Weber, et al. J. Phys. Chem. Lett. 9, 2838(2018).

Acknowledgments The authors were supported in part by the Grant-in-Aid for Scientific Research (Grant No. 15K05217) from the Japan Society for the Promotion of Science (JSPS), and a grant from Keio Gijuku Academic Development Funds. 